\title{
The effect of future ambient air pollution on human premature mortality to 2100 using output from the ACCMIP model ensemble
}

Raquel A. Silva et al.

Correspondence to: J. Jason West (jjwest@email.unc.edu)

The copyright of individual parts of the supplement might differ from the CC-BY 3.0 licence. 


\section{Air pollutant ambient concentrations}

The Atmospheric Chemistry and Climate Model Intercomparison Project (ACCMIP) included contributions from several modeling groups. While up to 14 models reported ozone concentrations (depending on the scenario), only up to 6 models reported species used in the calculation of $\mathrm{PM}_{2.5}$ concentrations, and up to 4 models reported their own estimate of $\mathrm{PM}_{2.5}$ concentrations (Table S1).

Figure S1 shows ten world regions used for all regional calculations presented below.

Global population-weighted differences (Future year - 2000) in ozone and $\mathrm{PM}_{2.5}$ concentrations for the different models are shown in Tables S2 and S3, respectively, while regional multi-model average differences are shown in Figures S2 and S3. For the global burden calculations, we use 1850 air pollutant concentrations reported by each model as counterfactual; for reference, we show the multi-model average concentrations in each grid cell (Figures S4 and S5).

For both pollutants, metrics are consistent with the underlying epidemiological studies for the health impact assessment:

- Seasonal (6-month) average of daily 1-hr maximum ozone concentration;

- Annual average $\mathrm{PM}_{2.5}$ concentration.

$\mathrm{PM}_{2.5}$ concentration is estimated using the sum of $\mathrm{PM}_{2.5}$ species mass mixing ratios reported by six models:

$$
\text { Estimated PM2.5=BC + OA + SO4 + SOA + NO3 + NH4 + 0.25*SS + 0.1*Dust, }
$$

where BC - Black Carbon, Dust, OA - (Primary) Organic Aerosol corrected to include species other than carbon, SO4 - Sulfate, SOA - Secondary Organic Aerosol, and SS - Sea Salt, 
following Fiore et al. (2012) and Silva et al. (2013). The factors 0.25 and 0.1 are intended to indicate the fractions of sea salt and dust that are in the $\mathrm{PM}_{2.5}$ size fraction.

\section{Population and Baseline Mortality Rates}

Table S4 includes present-day estimates of baseline mortality rates for cardiovascular diseases, chronic respiratory diseases and neoplasms given by IF projections for 2010 and GBD 2010.

Figure S6 shows future total and exposed population in 2030, 2050 and 2100 estimated from International Futures (IFs) country-level population per age group, used in the health impact assessment, as well as United Nations (UN) and Representative Concentration Pathway scenarios (RCPs) totals as context.

Figure S7 shows baseline mortality rates for chronic Respiratory diseases (RESP, ICD-9 ${ }^{1}$ BTL: B347), ischemic heart disease (IHD, ICD-9: 410-414), cerebrovascular disease (STROKE, ICD-9: 430-435, 437.0-437.2, 437.5-437.8), chronic obstructive pulmonary disease (COPD, ICD-9: 490-492.8, 494, 496) and lung cancer (LC, ICD-9 BTL: B101) estimated from IFs country-level mortality rates of cardiovascular diseases, chronic respiratory diseases and malignant neoplasms. Here we show average values for the exposed population (adults age 25 and older), but we used age distributed values for IHD and STROKE in the premature mortality calculation to align with available relative risks of exposure for these diseases.

\footnotetext{
${ }^{1}$ ICD-9 - International Classification of Diseases, revision 9.
} 


\section{Detailed results}

Table S5 shows the multi-model average global future ozone premature mortality, including uncertainty for RCP8.5, while Table S6 shows the multi-model average across ten world regions. Table S7 shows the multi-model average global $\mathrm{PM}_{2.5}$ mortality (IHD+STROKE+COPD+LC), including uncertainty for RCP8.5, while Table S8 shows the multi-model average across ten world regions. The multi-model average corresponds to the average of estimates given by the available models for each scenario/period. Figures S8 and S9 show the coefficient of variation in each grid cell of future air pollution-related premature mortality for all RCP scenarios in 2030, 2050 and 2100. Figures S10 and S11 show future global and regional air pollution-related premature mortality per million people in 2030, 2050 and 2100, for all RCPs relative to 2000 .

Tables S9 and S10 show the global burden on mortality of ozone and $\mathrm{PM}_{2.5}$ concentrations in 2000 relative to 1850, using present-day population and baseline mortality rates, and in 2030, 2050 and 2100 for all RCPs relative to 1850, using future population and baseline mortality rates. Also shown are two alternative cases for global burden calculation, using: A) 2000 concentrations relative to 1850 and present-day population but future baseline mortality rates; B) 2000 concentrations relative to 1850 but future population and baseline mortality rates. 


\section{References}

Cameron-Smith, P., Lamarque, J.-F., Connell, P., Chuang, C., and Vitt F.: Toward an Earth system model: atmospheric chemistry, coupling, and petascale computing, J. Phys.: Conf. Ser. 46, 343-350, doi:10.1088/1742-6596/46/1/048, 2006.

Collins, W. J., Bellouin, N., Doutriaux-Boucher, M., Gedney, N., Halloran, P., Hinton, T., Hughes, J., Jones, C. D., Joshi, M., Liddicoat, S., Martin, G., O'Connor, F., Rae, J., Senior, C., Sitch, S., Totterdell, I., Wiltshire, A. and Woodward, S.: Development and evaluation of an Earth-System model-HadGEM2, Geosci Model Dev, 4, 1051-75, 2011.

Donner, L. J., Wyman, B. L., Hemler, R. S., Horowitz, L. W., Ming, Y., Zhao, M., Golaz, J. C., Ginoux, P., Lin, S. J., Schwarzkopf, M. D., Austin, J., Alaka, G., Cooke, W. F., Delworth, T. L., Freidenreich, S. M., Gordon, C. T., Griffies, S. M., Held, I. M., Hurlin, W. J., Klein, S. A., Knutson, T. R., Langenhorst, A. R., Lee, H. C., Lin, Y. L., Magi, B. I., Malyshev, S. L., Milly, P. C. D., Naik, V., Nath, M. J., Pincus, R., Ploshay, J. J., Ramaswamy, V., Seman, C. J., Shevliakova, E., Sirutis, J. J., Stern, W. F., Stouffer, R. J., Wilson, R. J., Winton, M., Wittenberg, A. T. and Zeng, F. R.: The Dynamical Core, Physical Parameterizations, and Basic Simulation Characteristics of the Atmospheric Component AM3 of the GFDL Global Coupled Model CM3, J Climate, 24, 3484-519, 2011.

Fiore, A. M., Naik, V., Spracklen, D. V., Steiner, A., Unger, N., Prather, M., Bergmann, D., Cameron-Smith, P. J., Cionni, I., Collins, W. J., Dalsøren, S., Eyring, V., Folberth, G. a., Ginoux, P., Horowitz, L. W., Josse, B., Lamarque, J.-F., MacKenzie, I. a., Nagashima, T., O’Connor, F. M., Righi, M., Rumbold, S. T., Shindell, D. T., Skeie, R. B., Sudo, K., Szopa, S., Takemura, T. and Zeng, G.: Global air quality and climate, Chem Soc Rev, 41(19), 6663, doi:10.1039/c2cs35095e, 2012.

Institute for Health Metrics and Evaluation (IHME): Global Burden of Disease Study 2010 (GBD 2010) Results by Cause 1990-2010 - Country Level, Seattle, United States, 2013.

Jöckel, P., Tost, H., Pozzer, A., Bruhl, C., Buchholz, J., Ganzeveld, L., Hoor, P., Kerkweg, A., Lawrence, M. G., Sander, R., Steil, B., Stiller, G., Tanarhte, M., Taraborrelli, D., Van Aardenne, J. and Lelieveld., J: The atmospheric chemistry general circulation model ECHAM5/MESSy1: consistent simulation of ozone from the surface to the mesosphere, Atmos Chem Phys, 6, 5067104, 2006.

Josse, B., Simon, P., and Peuch, V.-H.: Rn-222 global simulations with the multiscale CTM MOCAGE, Tellus, 56B, 339-356, 2004.

Koch D., Schmidt G. A. and Field C. V.: Sulfur, sea salt, and radionuclide aerosols in GISS ModelE, J Geophys Res, 111, D06206, 2006.

Lamarque J.-F., Kyle G. P., Meinshausen M., Riahi K., Smith S. J., van Vuuren D. P., Conley A. J. and Vitt F.: Global and regional evolution of short-lived radiatively-active gases and aerosols in the Representative Concentration Pathways, Clim Change, 109, 191-212, 2011. 
Lamarque, J.-F., Shindell, D. T., Josse, B., Young, P. J., Cionni, I., Eyring, V., Bergmann, D., Cameron-Smith, P., Collins, W. J., Doherty, R., Dalsoren, S., Faluvegi, G., Folberth, G., Ghan, S. J., Horowitz, L. W., Lee, Y. H., MacKenzie, I. A., Nagashima, T., Naik, V., Plummer, D., Righi, M., Rumbold, S. T., Schulz, M., Skeie, R. B., Stevenson, D. S., Strode, S., Sudo, K., Szopa, S., Voulgarakis, A., and Zeng, G.: The Atmospheric Chemistry and Climate Model Intercomparison Project (ACCMIP): overview and description of models, simulations and climate diagnostics, Geosci. Model Dev., 6, 179-206, doi:10.5194/gmd-6-179-2013, 2013.

Naik, V., Horowitz, L. W., Fiore, A. M., Ginoux, P., Mao, J., Aghedo, A. M. and Levy, H.: Impact of preindustrial to present-day changes in short-lived pollutant emissions on atmospheric composition and climate forcing, J Geophys Res Atmos, 118, 8086-8110, doi:10.1002/jgrd.50608, 2013.

Oman L. D., Ziemke J. R., Douglass A. R., Waugh D. W., Lang C., Rodriguez J. M. and Nielsen J. E.: The response of tropical tropospheric ozone to ENSO, Geophys Res Lett, 38, 2011.

Righi, M., Eyring, V., Gottschaldt, K.-D., Klinger, C., Frank, F., Jöckel, P. and Cionni, I.: Quantitative evaluation of ozone and selected climate parameters in a set of EMAC simulations, Geosci. Model Dev., 8, 733-768, doi:10.5194/gmd-8-733-2015, 2015.

Scinocca J. F., McFarlane N. A., Lazare M., Li J. and Plummer D.: Technical Note: The CCCma third generation AGCM and its extension into the middle atmosphere, Atmos Chem Phys, 8, 7055-74, 2008.

Shindell D. T., Pechony O., Voulgarakis A., Faluvegi G., Nazarenko L., Lamarque J-F, Bowman K., Milly G., Kovari B., Ruedy R., Schmidt, G. A.: Interactive ozone and methane chemistry in GISS-E2 historical and future climate simulations, Atmos Chem Phys, 13, 2653-89, 2013.

Silva, R. A., West, J. J., Zhang, Y., Anenberg, S. C., Lamarque, J.-F., Shindell, D. T., Collins, W. J., Dalsoren, S., Faluvegi, G., Folberth, G., Horowitz, L. W., Nagashima, T., Naik, V., Rumbold, S., Skeie, R., Sudo, K., Takemura, T., Bergmann, D., Cameron-Smith, P., Cionni, I., Doherty, R. M., Eyring, V., Josse, B., MacKenzie, I. A., Plummer, D., Righi, M., Stevenson, D. S., Strode, S., Szopa, S. and Zeng, G.: Global premature mortality due to anthropogenic outdoor air pollution and the contribution of past climate change, Environ Res Lett, 8, 034005, doi:10.1088/1748-9326/8/3/034005, 2013.

Skeie R. B., Berntsen T. K., Myhre G., Tanaka K., Kvalevag M. M. and Hoyle C. R.: Anthropogenic radiative forcing time series from pre-industrial times until 2010, Atmos Chem Phys, 11, 11827-57, 2011.

Stevenson D. S., Doherty R. M., Sanderson M. G., Collins W. J., Johnson C. E. and Derwent R. G.: Radiative forcing from aircraft NOx emissions: Mechanisms and seasonal dependence, J Geophys Res, 109, 2004.

Szopa S., Balkanski Y., Schulz M., Bekki S., Cugnet D., Fortems-Cheiney A., Turquety S., Cozic A., Deandreis C., Hauglustaine D., Idelkadi A., Lathiere J., Lefevre F., Marchand M., 
Vuolo R., Yan N. and Dufresne J.-L.: Aerosol and ozone changes as forcing for climate evolution between 1850 and 2100, Climate Dynamics, doi: 10.1007/s00382-012-1408-y, 2012.

Teyssedre H., Michou M., Clark H. L., Josse B., Karcher F., Olivie D., Peuch V. H., SaintMartin D., Cariolle D., Attie J. L., Nedelec P., Ricaud P., Thouret V., Van Der A. R. J., VolzThomas A. and Cheroux F.: A new tropospheric and stratospheric Chemistry and Transport Model MOCAGE-Climat for multi-year studies: evaluation of the present-day climatology and sensitivity to surface processes, Atmos Chem Phys, 7, 5815-60, 2007.

Van Vuuren, D. P., Edmonds, J., Kainuma, M., Riahi, K., Thomson, A., Hibbard, K., Rose, S. K.: The representative concentration pathways: An overview, Clim Change, 109, 5-31, doi:10.1007/s10584-011-0148-z, 2011.

Watanabe S., Hajima T., Sudo K., Nagashima T., Takemura T., Okajima H., Nozawa T., Kawase H., Abe M., Yokohata T., Ise T., Sato H., Kato E., Takata K., Emori S. and Kawamiya M.: MIROC-ESM 2010: model description and basic results of CMIP5-20c3m experiments, Geosci Model Dev, 4, 845-72, 2011.

Zeng G., Pyle J. A. and Young P. J.: Impact of climate change on tropospheric ozone and its global budgets, Atmos Chem Phys, 8, 369-87, 2008.

Zeng G., Morgenstern O., Braesicke P. and Pyle J. A.: Impact of stratospheric ozone recovery on tropospheric ozone and its budget, Geophys Res Lett, 37, L09805, 2010. 


\section{Tables and Figures}

Table S1 - Models that reported ozone, $\mathrm{PM}_{2.5}$ species and $\mathrm{PM}_{2.5}$ (mmrpm2p5) concentrations for ACCMIP, with type of ozone output ( $\mathrm{h}$ - hourly, $\mathrm{m}$ - monthly) and number of reported $\mathrm{PM}_{2.5}$ species.

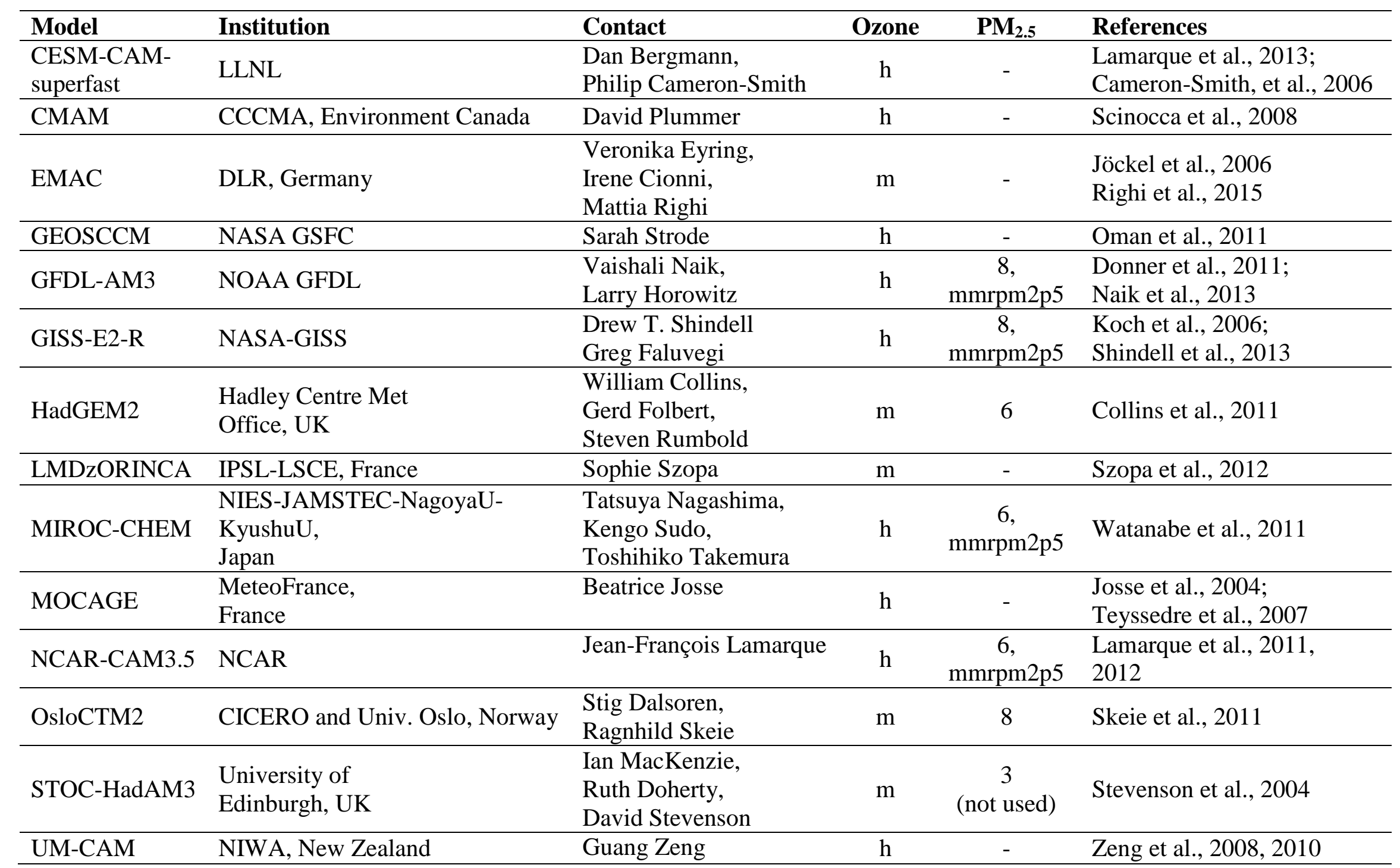


Table S2 - Global population-weighted differences (Future year - Hist. 2000) in ozone concentrations (ppb) for the 14 models in 2030, 2050, 2100 for the four RCPs. Pollutant concentrations are weighted by exposed population (adults aged 25 and older) in each future year. Models with the symbol * reported only monthly average ozone concentrations.

\begin{tabular}{|c|c|c|c|c|c|c|c|c|c|c|c|c|}
\hline \multirow{2}{*}{ Models } & \multicolumn{4}{|c|}{2030} & \multicolumn{4}{|c|}{2050} & \multicolumn{4}{|c|}{2100} \\
\hline & RCP2.6 & RCP4.5 & RCP6.0 & RCP8.5 & RCP2.6 & RCP4.5 & RCP6.0 & RCP8.5 & RCP2.6 & RCP4.5 & RCP6.0 & RCP8.5 \\
\hline $\begin{array}{l}\text { CESM-CAM- } \\
\text { superfast( }\left(^{\mathrm{a}}\right)\end{array}$ & & & & 3.7 & & & & & & & & 9.7 \\
\hline CMAM & -1.2 & 2.1 & & 4.4 & & & & & -9.0 & -6.1 & & 9.6 \\
\hline GEOSCCM & & & & & & & & & & & -4.7 & \\
\hline GFDL-AM3 & 0.5 & 4.7 & 1.3 & 9.0 & -1.3 & 1.6 & 2.3 & 5.7 & -8.7 & -9.6 & -8.3 & 5.1 \\
\hline GISS-E2-R & 0.2 & 4.2 & 1.5 & 12.0 & 0.1 & 0.2 & 2.9 & 5.6 & -5.1 & -11.7 & -7.6 & 2.9 \\
\hline MIROC-CHEM & -0.5 & & 0.9 & 6.9 & -2.3 & & 1.6 & 4.1 & -8.1 & & -8.5 & 1.3 \\
\hline MOCAGE & 2.7 & & 1.5 & 15.2 & & & & & -9.4 & & -11.9 & 1.5 \\
\hline NCAR-CAM3.5 & -2.5 & 0.9 & -2.1 & 3.7 & & & & & -11.7 & -11.2 & -11.4 & 0.9 \\
\hline UM-CAM & -1.4 & 2.5 & & 7.1 & & & & & -8.9 & -7.3 & & 3.9 \\
\hline CICERO-OsloCTM2* & 0.0 & 2.8 & & 8.2 & & & & & -9.3 & -9.5 & & 4.2 \\
\hline EMAC* & & 4.0 & & 9.5 & & & & & & -8.9 & & 5.9 \\
\hline HadGEM2* & -0.9 & -0.1 & & 0.5 & & & & & -7.7 & -3.7 & & 13.6 \\
\hline LMDzORINCA* & -1.7 & 1.7 & 0.2 & 7.2 & -8.7 & -4.7 & -2.8 & 0.7 & -9.9 & -9.0 & -8.9 & 3.6 \\
\hline STOC-HadAM3* & 0.7 & & & 11.0 & & & & & -10.3 & & & 3.5 \\
\hline
\end{tabular}

( $\left.{ }^{\mathrm{a}}\right)$ CESM-CAM-superfast reported concentrations for RCP2.6 and RCP 6.0, but the simulations for these scenarios used an inconsistent SST file and are not a matched set with the other simulations, so they were not considered here. 
Table S3 - Global population-weighted differences (Future year - Hist. 2000) in $\mathrm{PM}_{2.5}$ concentrations (estimated as a sum of reported species) $\left(\mu \mathrm{g} / \mathrm{m}^{3}\right)$ for the 6 models in 2030, 2050, 2100 for the four RCPs. Pollutant concentrations are weighted by exposed population (adults aged 25 and older) in each future year.

\begin{tabular}{|c|c|c|c|c|c|c|c|c|c|c|c|c|}
\hline \multirow{2}{*}{ Models } & \multicolumn{4}{|c|}{2030} & \multicolumn{4}{|c|}{2050} & \multicolumn{4}{|c|}{2100} \\
\hline & RCP2.6 & RCP4.5 & RCP6.0 & RCP8.5 & RCP2.6 & RCP4.5 & RCP6.0 & RCP8.5 & RCP2.6 & RCP4.5 & RCP6.0 & RCP8.5 \\
\hline GFDL-AM3 & 0.1 & 1.1 & 0.03 & 1.9 & -3.4 & -2.3 & 1.4 & -1.0 & -5.6 & -6.3 & -4.8 & -3.3 \\
\hline GISS-E2-R & -2.0 & -1.3 & 0.9 & 0.1 & -4.4 & -4.5 & 0.8 & -3.1 & -5.1 & -5.9 & -5.1 & -4.0 \\
\hline NCAR-CAM3.5 & -0.4 & 0.01 & -0.03 & 1.3 & & & & & -5.7 & -6.4 & -4.9 & -3.9 \\
\hline MIROC-CHEM & 0.2 & & 0.5 & 1.0 & -2.7 & & 1.3 & -1.5 & -3.9 & & -3.3 & -2.1 \\
\hline CICERO-OsloCTM2 & 2.6 & & & 3.8 & & & & & -3.3 & & & -0.4 \\
\hline HadGEM2 & 0.5 & 0.9 & & 1.7 & & & & & -3.5 & -4.6 & & -3.9 \\
\hline
\end{tabular}

Table S4 - IF projections for 2010 and GBD 2010 estimates of age-standardized mortality rates (deaths per 100,000 people).

\begin{tabular}{l|l|l}
\hline \multicolumn{1}{c|}{ Diseases } & \multicolumn{1}{c|}{ IF } & \multicolumn{1}{c}{ GBD 2010 } \\
\hline Cardiovascular & 234.9 & 234.8 \\
\hline Chronic Respiratory & 58.4 & 57.0 \\
\hline Neoplasms & 106.9 & 121.4 \\
\hline
\end{tabular}


Table S5 - Change in global respiratory premature ozone mortality in 2030, 2050 and 2100 for all RCPs (considering the change in future ozone concentrations relative to 2000 concentrations), showing the multi-model average (deaths/year) for RCP2.6, RCP4.5 and RCP6.0 deterministic estimates and the empirical mean with 95\% CI in parenthesis for RCP8.5 probabilistic estimates (including uncertainty in the RRs and across models). These results correspond to Figure 1. All numbers are rounded to three significant digits.

2030

\begin{tabular}{lrrr}
\hline RCP2.6 & 11,900 & & \\
RCP4.5 & 100,000 & & \\
RCP6 & 71,200 & & \\
RCP8.5 & 264,000 & $(-39,300$, & $648,000)$ \\
\hline \multicolumn{5}{c}{$\mathbf{2 0 5 0}$} \\
\hline RCP2.6 & $-450,000$ & & \\
RCP4.5 & $-360,000$ & & \\
RCP6 & 441,000 & & $556,000)$ \\
RCP8.5 & 246,000 & $(-59,600$, & \\
\hline & & 2100 & \\
RCP2.6 & $-1,020,000$ & & \\
RCP4.5 & $-917,000$ & & \\
RCP6 & $-718,000$ & & \\
RCP8.5 & 316,000 & $(-187,000,380,000)$ \\
\hline
\end{tabular}

Table S6 - Premature ozone-related respiratory mortality in ten world regions relative to 2000 concentrations: (a) 2030, (b) 2050, (c) 2100, showing the multi-model average (deaths/year) of the deterministic results. All numbers are rounded to three significant digits.

(a) 2030

\begin{tabular}{lrrrr}
\hline \multicolumn{1}{c}{ Region } & RCP2.6 & RCP4.5 & RCP6.0 & RCP8.5 \\
\hline North America & $-17,000$ & $-12,500$ & $-10,900$ & $-8,200$ \\
\hline South America & $-2,710$ & -500 & $-3,260$ & 1,840 \\
\hline Europe & $-8,870$ & $-5,590$ & $-7,190$ & -880 \\
\hline Former Soviet Union & $-2,200$ & $-1,030$ & $-1,600$ & 660 \\
\hline Africa & 2,100 & 6,440 & $-3,520$ & 9,020 \\
\hline India & 52,900 & 82,000 & $-6,440$ & 124,000 \\
\hline East Asia & $-11,300$ & 25,700 & 103,000 & 127,000 \\
\hline Southeast Asia & 2,980 & 5,010 & 4,890 & 5,980 \\
\hline Australia & -280 & -120 & -100 & 20 \\
\hline Middle East & $-3,630$ & 930 & $-3,700$ & 7,460 \\
\hline
\end{tabular}


(b) $\mathbf{2 0 5 0}$

\begin{tabular}{lrrrr}
\hline \multicolumn{1}{c}{ Region } & RCP2.6 & RCP4.5 & RCP6.0 & RCP8.5 \\
\hline North America & $-85,500$ & $-70,200$ & $-52,100$ & $-41,100$ \\
\hline South America & $-8,180$ & $-4,910$ & $-8,920$ & 8,530 \\
\hline Europe & $-49,400$ & $-40,000$ & $-34,800$ & $-16,600$ \\
\hline Former Soviet Union & $-9,760$ & $-6,390$ & $-5,710$ & -440 \\
\hline Africa & 13,100 & 16,600 & $-5,520$ & 30,500 \\
\hline India & 154,000 & 290,000 & 32,200 & 256,000 \\
\hline East Asia & $-439,000$ & $-514,000$ & 518,000 & 3,830 \\
\hline Southeast Asia & 900 & $-21,300$ & 19,600 & $-9,920$ \\
\hline Australia & $-1,260$ & -590 & -490 & 250 \\
\hline Middle East & $-24,800$ & $-9,930$ & $-21,100$ & 18,300 \\
\hline
\end{tabular}

(c) 2100

\begin{tabular}{lrrrr}
\hline \multicolumn{1}{c}{ Region } & RCP2.6 & RCP4.5 & RCP6.0 & RCP8.5 \\
\hline North America & $-104,000$ & $-66,300$ & $-111,000$ & $-21,100$ \\
\hline South America & $-19,800$ & $-20,200$ & $-25,900$ & 7,950 \\
\hline Europe & $-44,600$ & $-24,900$ & $-41,600$ & 2,390 \\
\hline Former Soviet Union & $-12,500$ & $-8,180$ & $-11,100$ & 1,290 \\
\hline Africa & 51,100 & $-16,000$ & $-49,400$ & 128,000 \\
\hline India & $-230,000$ & $-267,000$ & $-125,000$ & 292,000 \\
\hline East Asia & $-509,000$ & $-383,000$ & $-241,000$ & $-99,700$ \\
\hline Southeast Asia & $-65,000$ & $-71,400$ & $-28,000$ & $-21,000$ \\
\hline Australia & $-1,620$ & -990 & $-1,510$ & 790 \\
\hline Middle East & $-83,400$ & $-58,100$ & $-83,200$ & 29,800 \\
\hline
\end{tabular}


Table S7 - Change in global premature $\mathrm{PM}_{2.5}$ mortality (IHD+Stroke+COPD+LC) in 2030, 2050 and 2100 for all RCPs (considering the change in future $\mathrm{PM}_{2.5}$ concentrations relative to 2000 concentrations), showing multi-model average (deaths/year) for RCP2.6, RCP4.5 and RCP6.0 deterministic estimates and the empirical mean with 95\% CI in parenthesis for RCP8.5 probabilistic estimates (including uncertainty in the RRs and across models). These results correspond to Figure 4. All numbers are rounded to three significant digits.

2030

\begin{tabular}{lrrr}
\hline RCP2.6 & $-258,000$ \\
RCP4.5 & $-289,000$ \\
RCP6 & $-169,000$ & & \\
RCP8.5 & 17,200 & $(-386,000$, & $661,000)$ \\
\hline \multicolumn{4}{c}{$\mathbf{2 0 5 0}$} \\
\hline RCP2.6 & $-1,670,000$ \\
RCP4.5 & $-1,760,000$ \\
RCP6 & 16,700 \\
RCP8.5 & $-1,210,000$ & $(-1,730,000$, & $-835,000)$ \\
\hline \multicolumn{5}{c}{$\mathbf{2 1 0 0}$} \\
\hline RCP2.6 & $-1,930,000$ & \\
RCP4.5 & $-2,390,000$ & & \\
RCP6 & $-1,760,000$ & & \\
RCP8.5 & $-1,310,000$ & $(-2,040,000$, & $-174,000)$ \\
\hline
\end{tabular}

Table S8 - Premature $\mathrm{PM}_{2.5}$ mortality (IHD+Stroke+COPD+LC) in ten world regions: (a) 2030, (b) 2050, (c) 2100, showing the multi-model average (deaths/year) of the deterministic results. All numbers are rounded to three significant digits.

(a) $\quad 2030$

\begin{tabular}{lrrrr}
\hline \multicolumn{1}{c}{ Region } & RCP2.6 & RCP4.5 & RCP6.0 & RCP8.5 \\
\hline North America & $-77,800$ & $-83,500$ & $-59,700$ & $-77,100$ \\
\hline South America & -570 & $-6,100$ & $-6,960$ & $-6,290$ \\
\hline Europe & $-153,000$ & $-152,000$ & $-137,000$ & $-176,000$ \\
\hline Former Soviet Union & $-119,000$ & $-82,000$ & $-101,000$ & $-116,000$ \\
\hline Africa & 35,100 & 31,800 & $-10,200$ & 46,100 \\
\hline India & 150,000 & 176,000 & $-2,690$ & 245,000 \\
\hline East Asia & $-90,100$ & $-137,800$ & 151,000 & 86,000 \\
\hline Southeast Asia & 27,800 & $-30,700$ & 36,200 & -430 \\
\hline Australia & -560 & -180 & -440 & -30 \\
\hline Middle East & $-30,700$ & $-4,430$ & $-37,400$ & $-7,230$ \\
\hline
\end{tabular}


(b) $\mathbf{2 0 5 0}$

\begin{tabular}{lrrrr}
\hline \multicolumn{1}{c}{ Region } & RCP2.6 & RCP4.5 & RCP6.0 & RCP8.5 \\
\hline North America & $-106,000$ & $-114,000$ & $-104,000$ & $-107,000$ \\
\hline South America & $-7,550$ & $-9,550$ & $-6,720$ & $-7,940$ \\
\hline Europe & $-198,000$ & $-187,000$ & $-193,000$ & $-200,000$ \\
\hline Former Soviet Union & $-144,000$ & $-158,000$ & $-154,000$ & $-156,000$ \\
\hline Africa & 40,200 & 46,000 & $-21,100$ & 66,100 \\
\hline India & $-6,540$ & 97,000 & 152,000 & 308,000 \\
\hline East Asia & $-1,050,000$ & $-1,200,000$ & 356,000 & $-906,000$ \\
\hline Southeast Asia & $-113,000$ & $-193,000$ & 52,500 & $-182,000$ \\
\hline Australia & -370 & -390 & -250 & -240 \\
\hline Middle East & $-81,200$ & $-47,800$ & $-64,300$ & $-47,200$ \\
\hline
\end{tabular}

(c) 2100

\begin{tabular}{lrrrr}
\hline \multicolumn{1}{c}{ Region } & RCP2.6 & RCP4.5 & RCP6.0 & RCP8.5 \\
\hline North America & $-105,000$ & $-128,000$ & $-116,000$ & $-110,000$ \\
\hline South America & $-15,600$ & $-21,300$ & $-12,800$ & $-15,000$ \\
\hline Europe & $-104,000$ & $-110,000$ & $-112,000$ & $-103,000$ \\
\hline Former Soviet Union & $-75,200$ & $-109,000$ & $-111,000$ & $-97,500$ \\
\hline Africa & 111,000 & $-68,100$ & $-107,000$ & 147,000 \\
\hline India & $-531,000$ & $-606,000$ & $-315,000$ & 62,700 \\
\hline East Asia & $-886,000$ & $-926,000$ & $-673,000$ & $-882,000$ \\
\hline Southeast Asia & $-153,000$ & $-250,000$ & $-103,000$ & $-202,000$ \\
\hline Australia & 30 & -850 & -770 & -440 \\
\hline Middle East & $-168,000$ & $-176,000$ & $-209,000$ & $-127,000$ \\
\hline
\end{tabular}


Table S9 - Global burden on mortality of ozone concentrations in the present-day for 2000 concentrations relative to 1850, showing multi-model average and 95\% CI including uncertainty in RR and across models (deaths/year), and in 2030, 2050 and 2100 for all RCPs relative to 1850, showing multi-model averages (deaths/year) given by the deterministic values. Also shown, future burdens using (Case A) 2000 concentrations relative to 1850 and present-day population but future baseline mortality rates and (Case B) 2000 concentrations relative to 1850 but future population and baseline mortality rates. These results are plotted in Figure 7. All numbers are rounded to three significant digits.

\begin{tabular}{lrrrr}
\hline & $\mathbf{2 0 0 0}$ & $\mathbf{2 0 3 0}$ & $\mathbf{2 0 5 0}$ & $\mathbf{2 1 0 0}$ \\
\hline Present-day & 382,000 & & & \\
& $(121,000$ to 728,400$)$ & & & \\
\hline RCP2.6 & & 756,000 & $1,840,000$ & $1,170,000$ \\
\hline RCP4.5 & & 775,000 & $1,990,000$ & $1,090,000$ \\
\hline RCP6.0 & 891,000 & $2,600,000$ & $1,570,000$ \\
\hline RCP8.5 & 972,000 & $2,460,000$ & $2,360,000$ \\
\hline Case A & & 569,000 & $1,540,000$ & $1,490,000$ \\
\hline Case B & & 735,000 & $2,090,000$ & $2,040,000$ \\
\hline
\end{tabular}

Table S10 - Global burden on mortality of $\mathrm{PM}_{2.5}$ concentrations in the present-day for 2000 concentrations relative to 1850, showing multi-model average and 95\% CI including uncertainty in RR and across models (deaths/year), and in 2030, 2050 and 2100 for all RCPs relative to 1850 , showing multi-model averages (deaths/year) given by the deterministic values. Also shown, future burdens using (Case A) 2000 concentrations relative to 1850 and present-day population but future baseline mortality rates and (Case B) 2000 concentrations relative to 1850 but future population and baseline mortality rates. These results are plotted in Figure 8. All numbers are rounded to three significant digits.

\begin{tabular}{|c|c|c|c|c|}
\hline & 2000 & 2030 & 2050 & 2100 \\
\hline Present-day & $\begin{array}{r}1,700,000 \\
(1,300,000 \text { to } 2,100,000)\end{array}$ & & & \\
\hline RCP2.6 & & $2,360,000$ & $1,820,000$ & 948,000 \\
\hline RCP4.5 & & $2,440,000$ & $1,870,000$ & 559,000 \\
\hline RCP6.0 & & $2,640,000$ & $3,500,000$ & $1,350,00 c$ \\
\hline RCP8.5 & & $2,620,000$ & $2,250,000$ & $1,550,000$ \\
\hline Case A & & $1,590,000$ & $1,440,000$ & $1,230,000$ \\
\hline Case B & & $2,620,000$ & $3,310,000$ & $2,880,00 \mathrm{C}$ \\
\hline
\end{tabular}




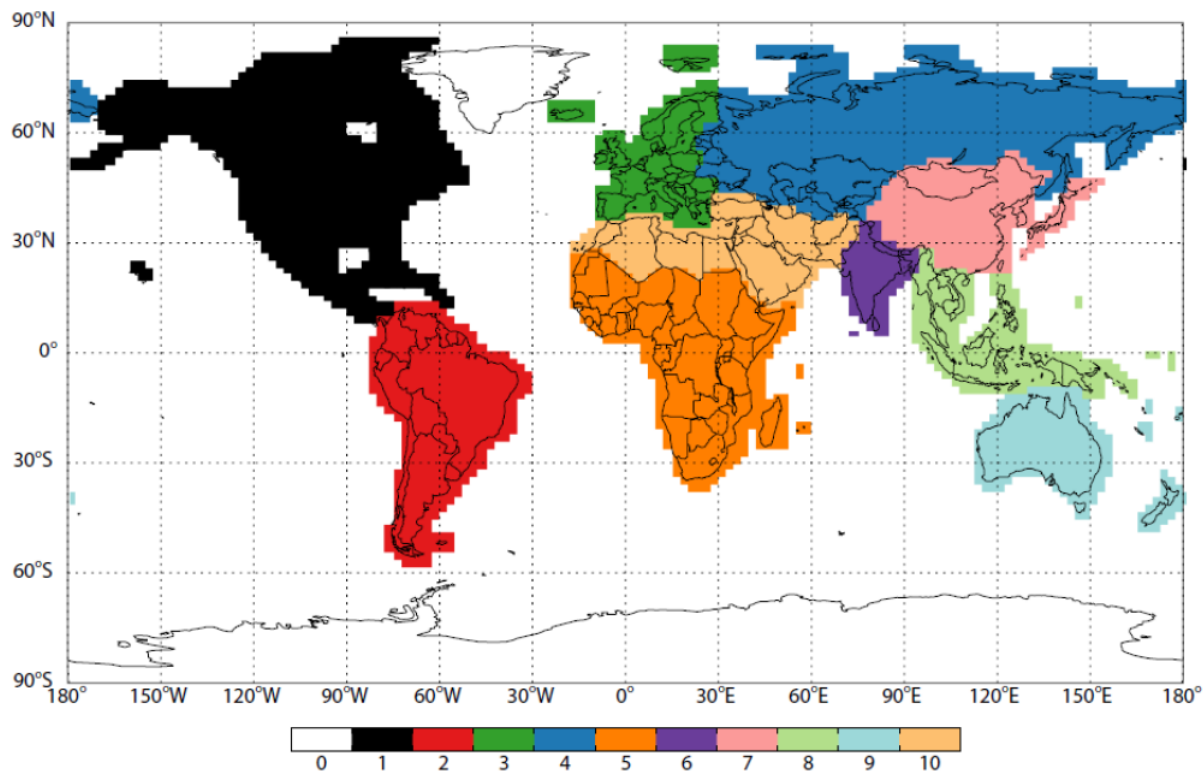

1 - North America

2 - South America

3 - Europe

4 - Former Soviet Union

5 - Africa

6 - India

7 - East Asia

8 - Southeast Asia

9 - Australia

10 - Middle East

Figure S1 - Ten world regions 


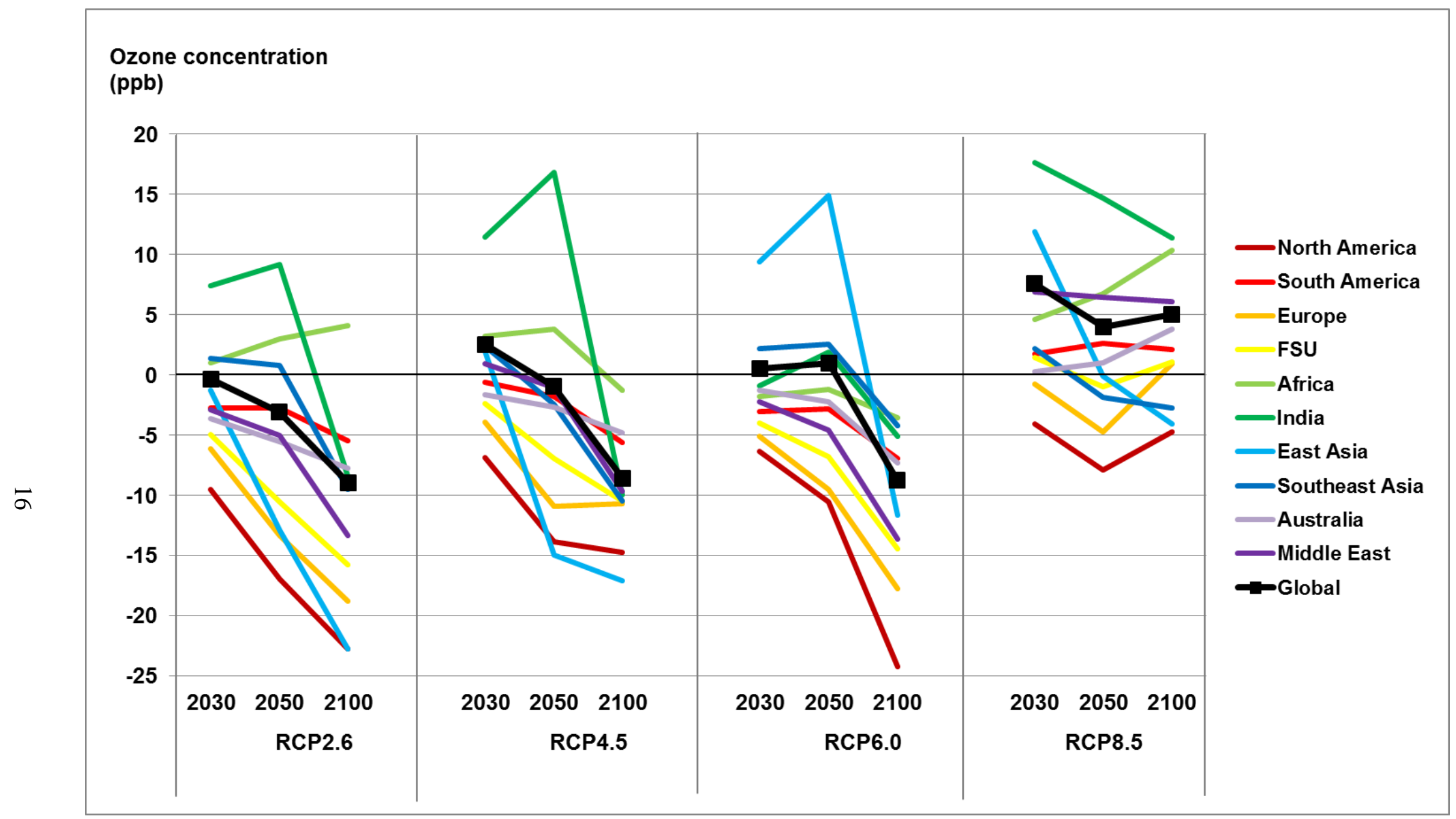

Figure S2 - Regional population-weighted difference in ozone concentrations (ppb) in 2030, 2050 and 2100 relative to 2000. (FSU Former Soviet Union) 


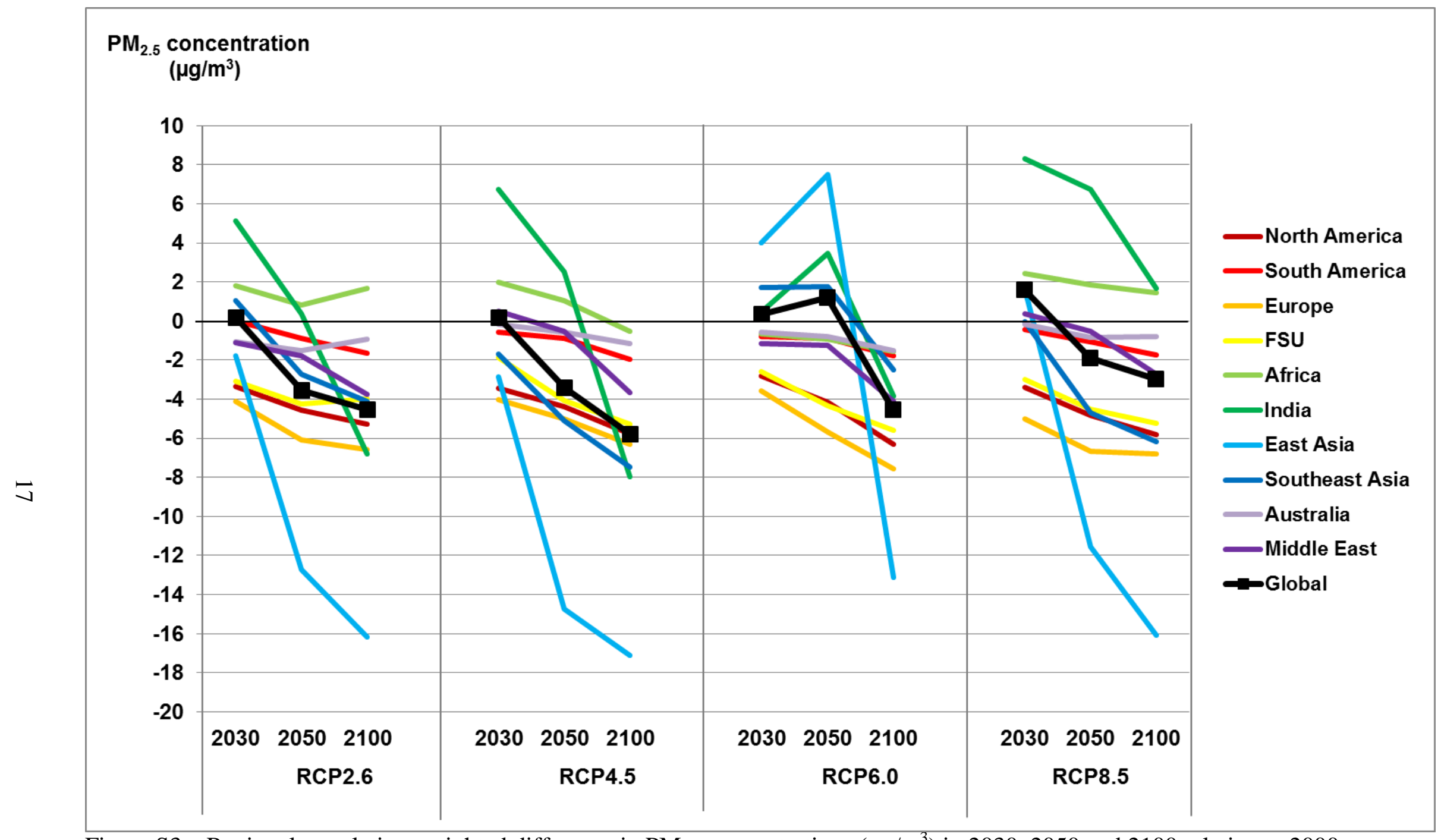

Figure S3 - Regional population-weighted difference in $\mathrm{PM}_{2.5}$ concentrations $\left(\mu \mathrm{g} / \mathrm{m}^{3}\right)$ in 2030, 2050 and 2100 relative to 2000. 


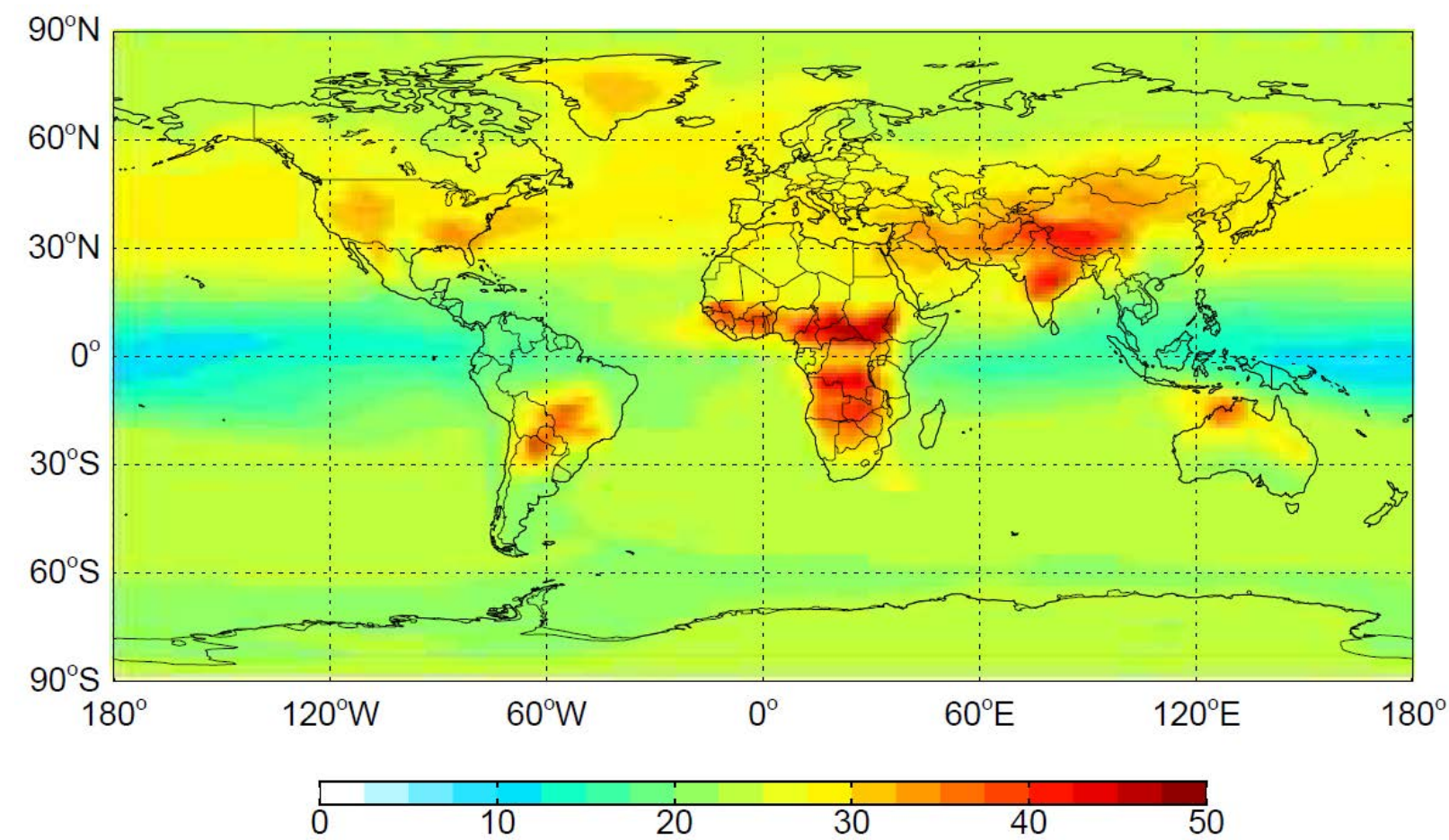

Figure S4 - Spatial distribution of ozone concentrations in 1850 (ppb), showing the multi-model mean in each grid cell.

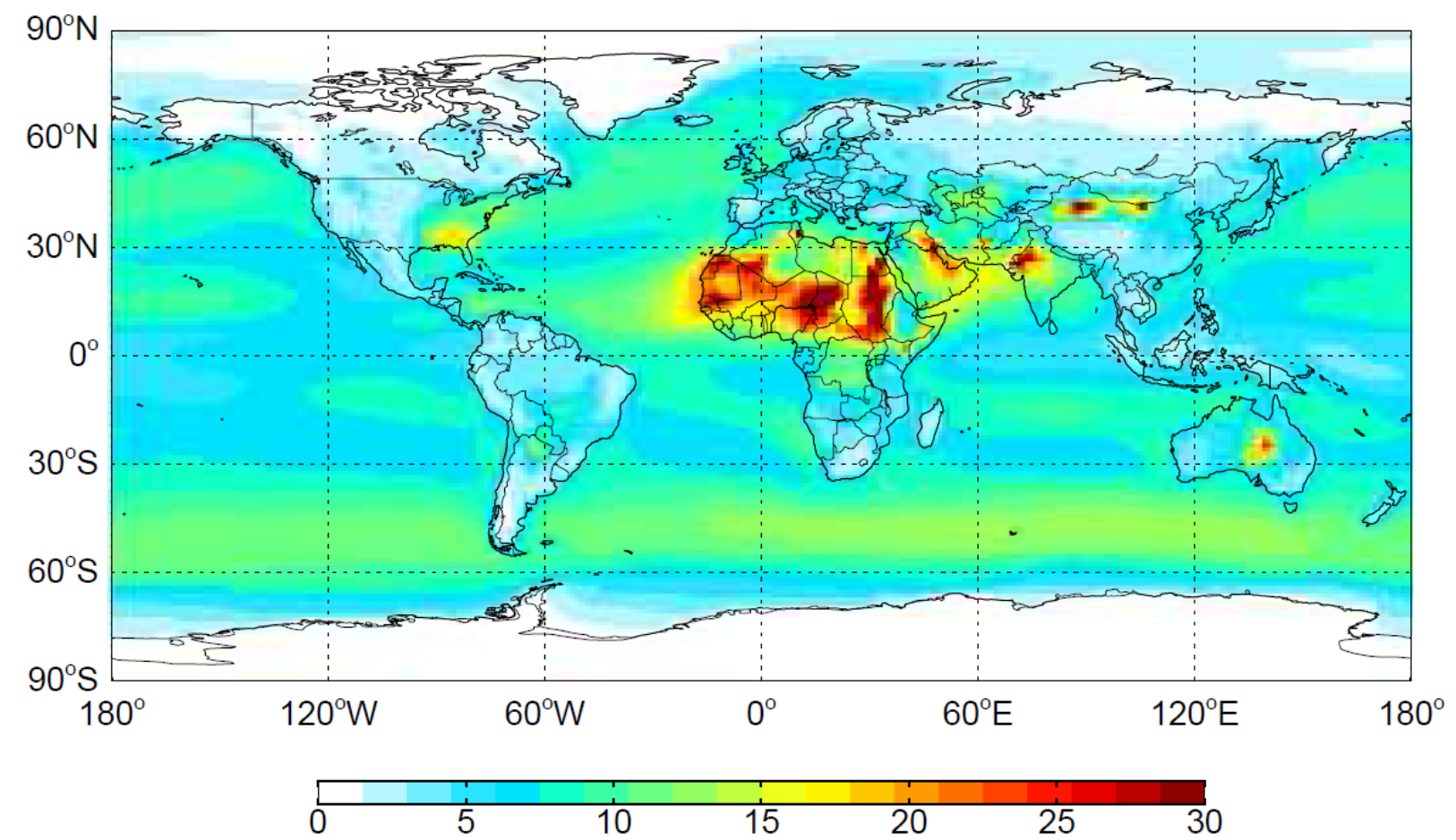

Figure S5 - Spatial distribution of $\mathrm{PM}_{2.5}$ concentrations (sum of species) in $1850\left(\mu \mathrm{g} / \mathrm{m}^{3}\right)$, showing the multi-model mean in each grid cell. 


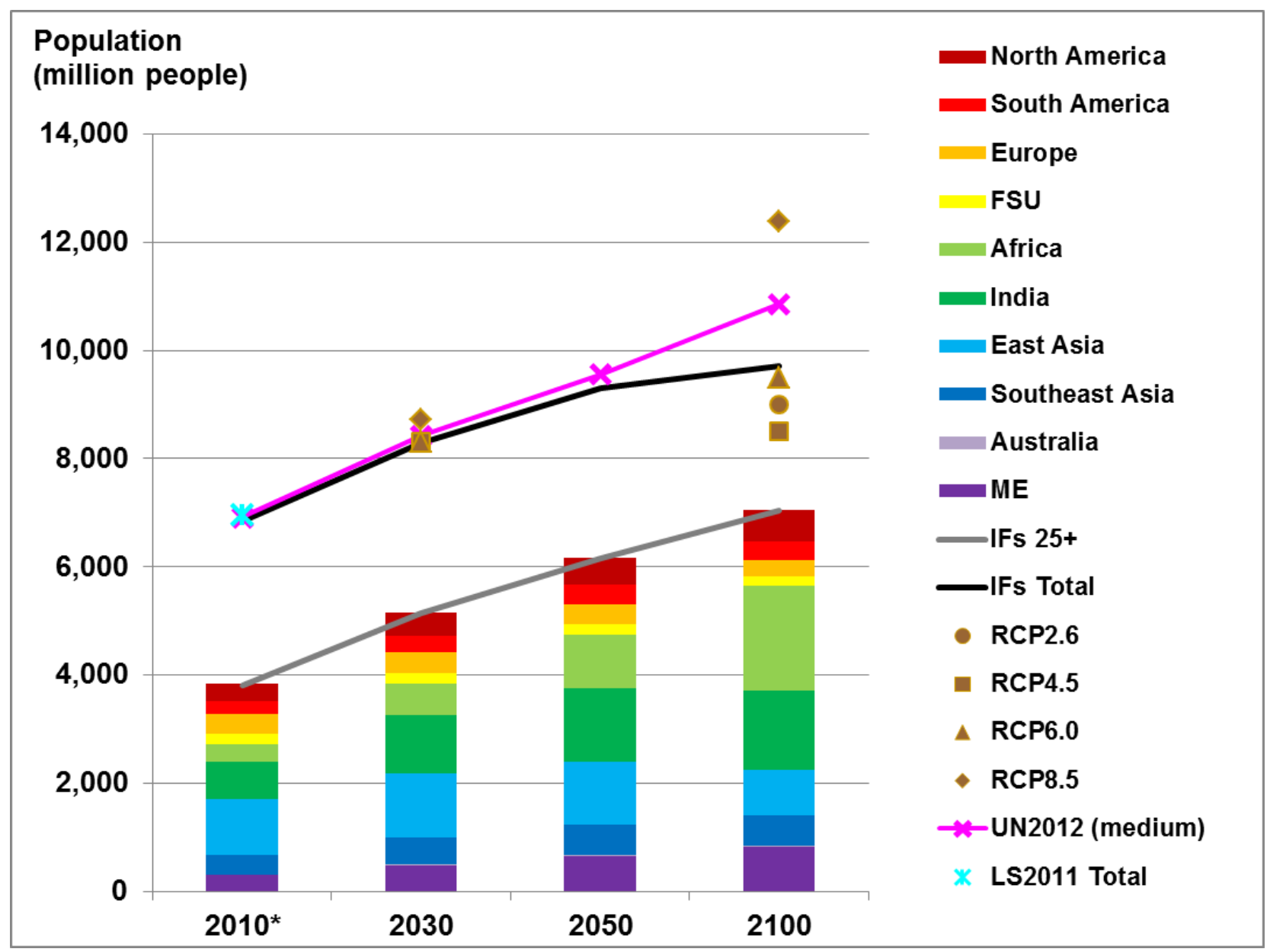

Figure S6 - Present-day and future population (millions of people) showing global totals for exposed population (adults 25 and older) from Landscan 2011 (2010) and IFs (2030, 2050, 2100), as well as total population for the RCP scenarios for 2030 and 2100 (Van Vuuren et al., 2011) and for UN Population Prospects 2012 medium fertility scenario for 2030, 2050 and 2100. Also shown are regional exposed populations for IFs.

Sources:

- Oak Ridge National Laboratory (ONRL) - LandScan 2011 Global Population Dataset, http://spruce.lib.unc.edu.libproxy.lib.unc.edu/content/gis/LandScan/. Data retrieved on 12/05/2012.

- $\quad$ Web-Based IFs - The International Futures (IFs) modeling system, version 6.54., www.ifs.du.edu. Data retrieved on 07/2012.

- United Nations, Department of Economic and Social Affairs, Population Division (2013). World Population Prospects: The 2012 Revision. http://esa.un.org/wpp/ExcelData/population.htm. Data retrieved on 12/03/2013. 


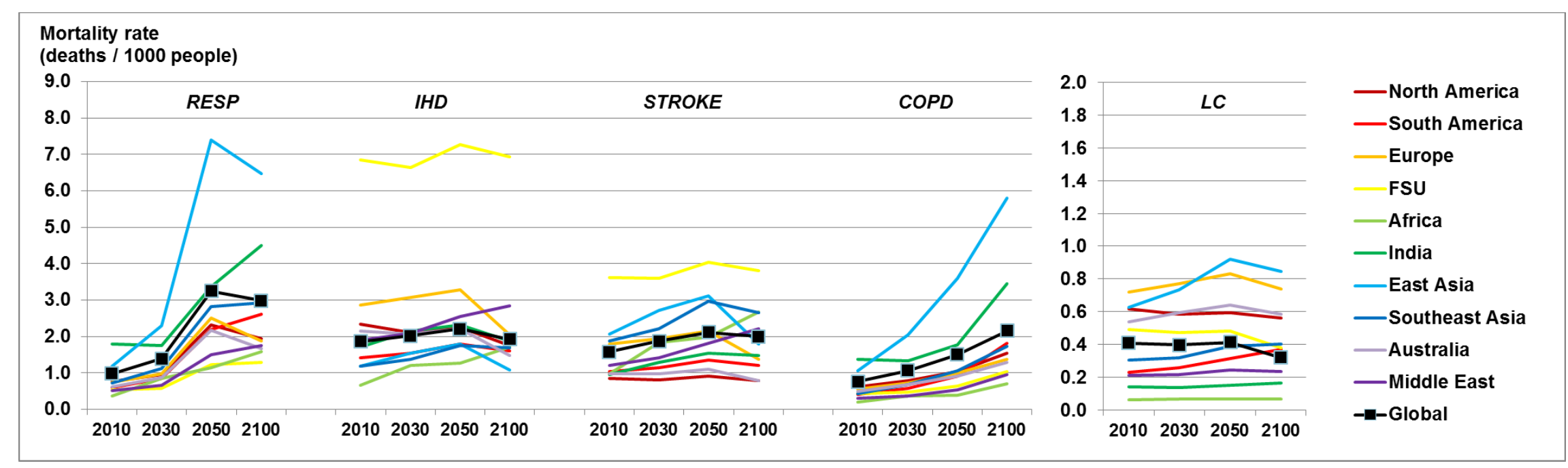

Figure S7 - Global and regional average present-day and future baseline mortality rates (deaths per 1000 people per year) for RESP, IHD, STROKE, COPD and LC, for adults aged 25 and older from the Global Burden of Disease Study 2010 mortality dataset (IHME, ऽ 2013) and IFs (2030, 2050, 2100). The IHD and Stroke averages are shown for illustration only, since the mortality estimates are obtained using baseline mortality rates per 5-year age group.

Sources:

- Web-Based IFs - The International Futures (IFs) modeling system, version 6.54., www.ifs.du.edu. Data retrieved on 07/2012.

- IHME (2013). Data retrieved from 12/2013 to 03/2014. 


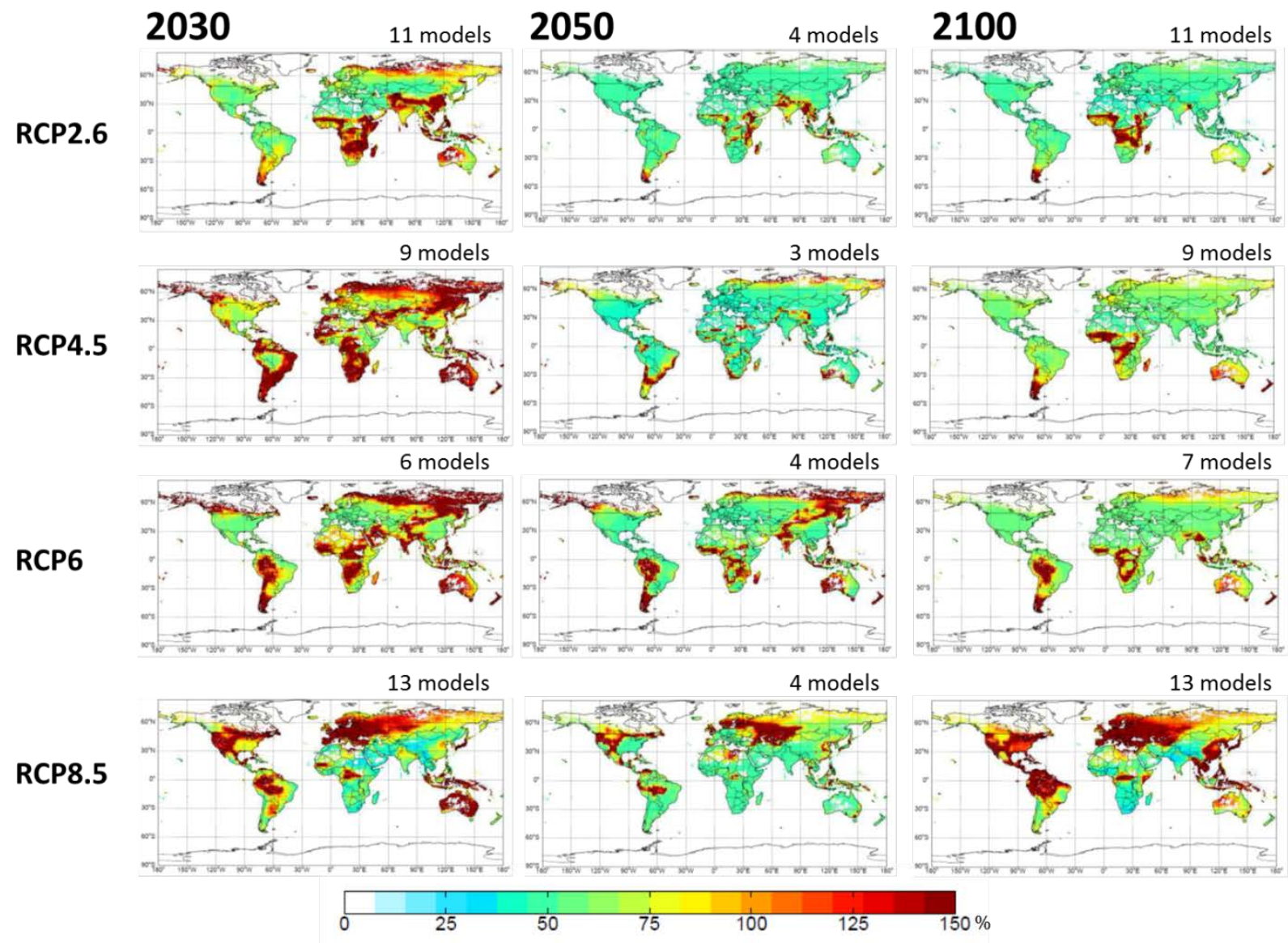

Figure S8 - Spatial distribution of model variability in future ozone respiratory mortality for all RCP scenarios in 2030, 2050 and 2100, showing the coefficient of variation of mortality estimates in each grid cell. 


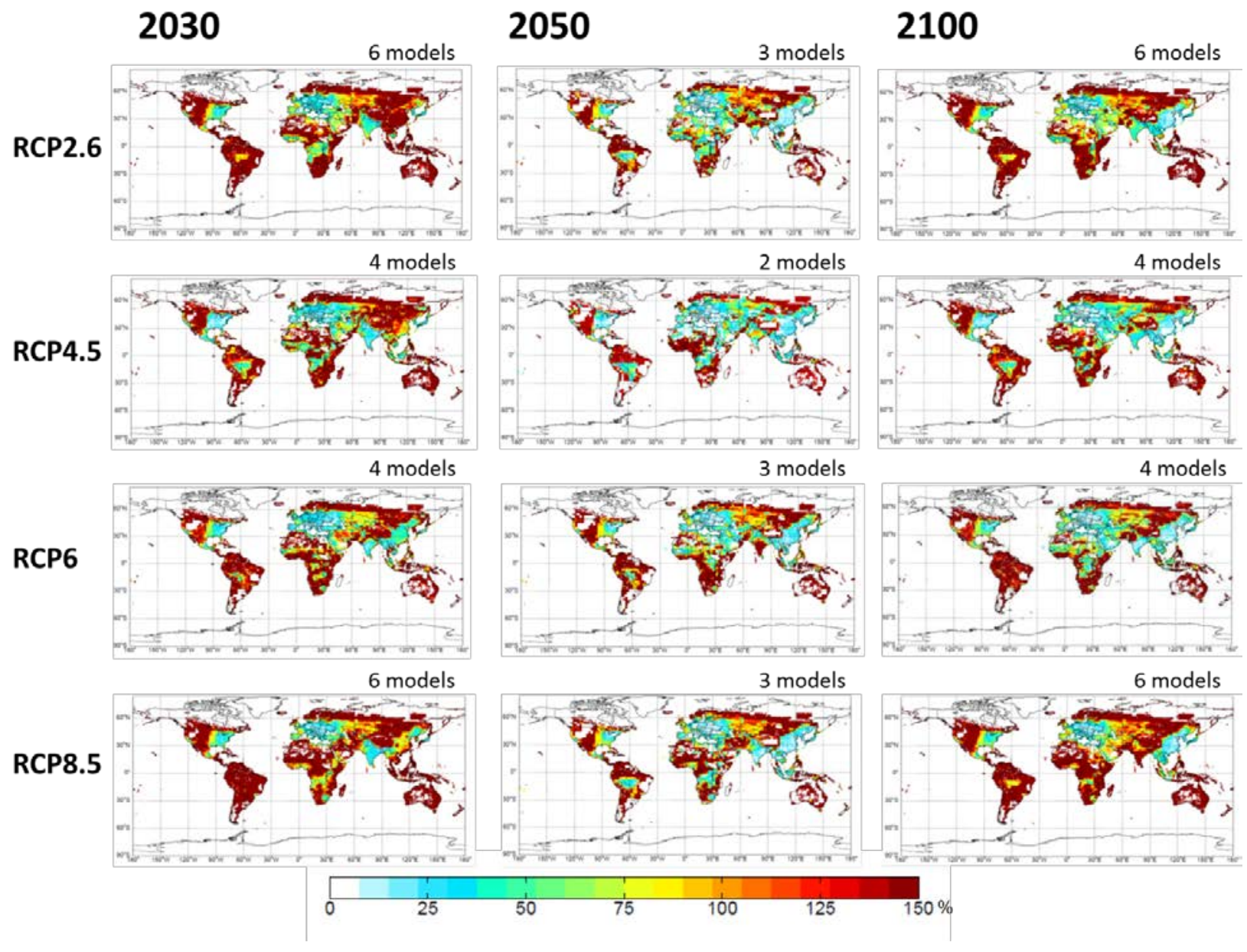

Figure S9 - Spatial distribution of model variability in future premature mortality (IHD+STROKE+COPD+LC) for $\mathrm{PM}_{2.5}$ calculated as a sum of species for all RCP scenarios in 2030, 2050 and 2100, showing the coefficient of variation of mortality estimates in each grid cell. 


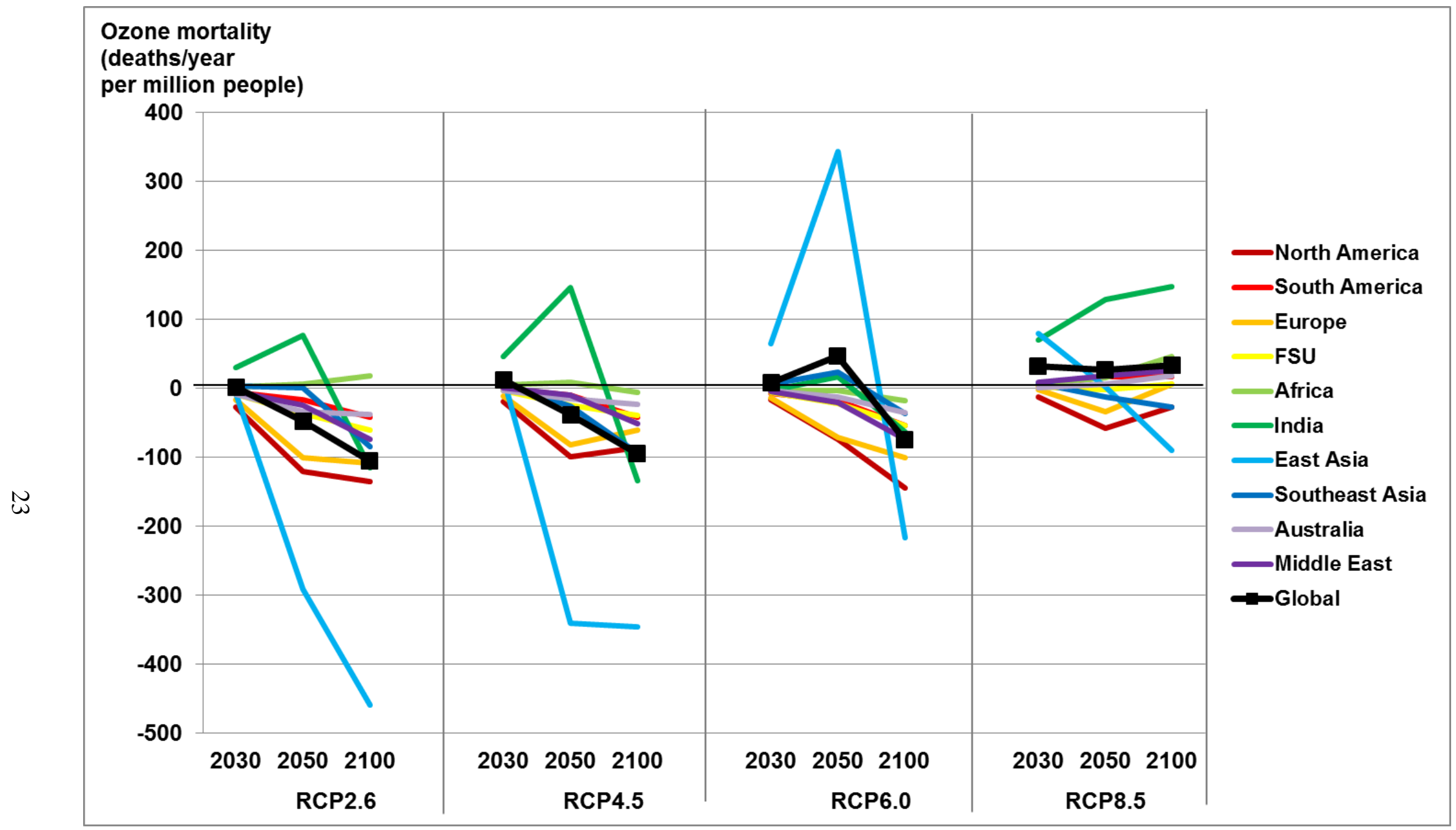

Figure S10 - Future ozone respiratory mortality per million people for all RCP scenarios in 2030, 2050 and 2100, showing the multimodel regional average (deaths/year per million people) in ten world regions (Figure S1) and globally, for future air pollutant concentrations relative to 2000 concentrations. 


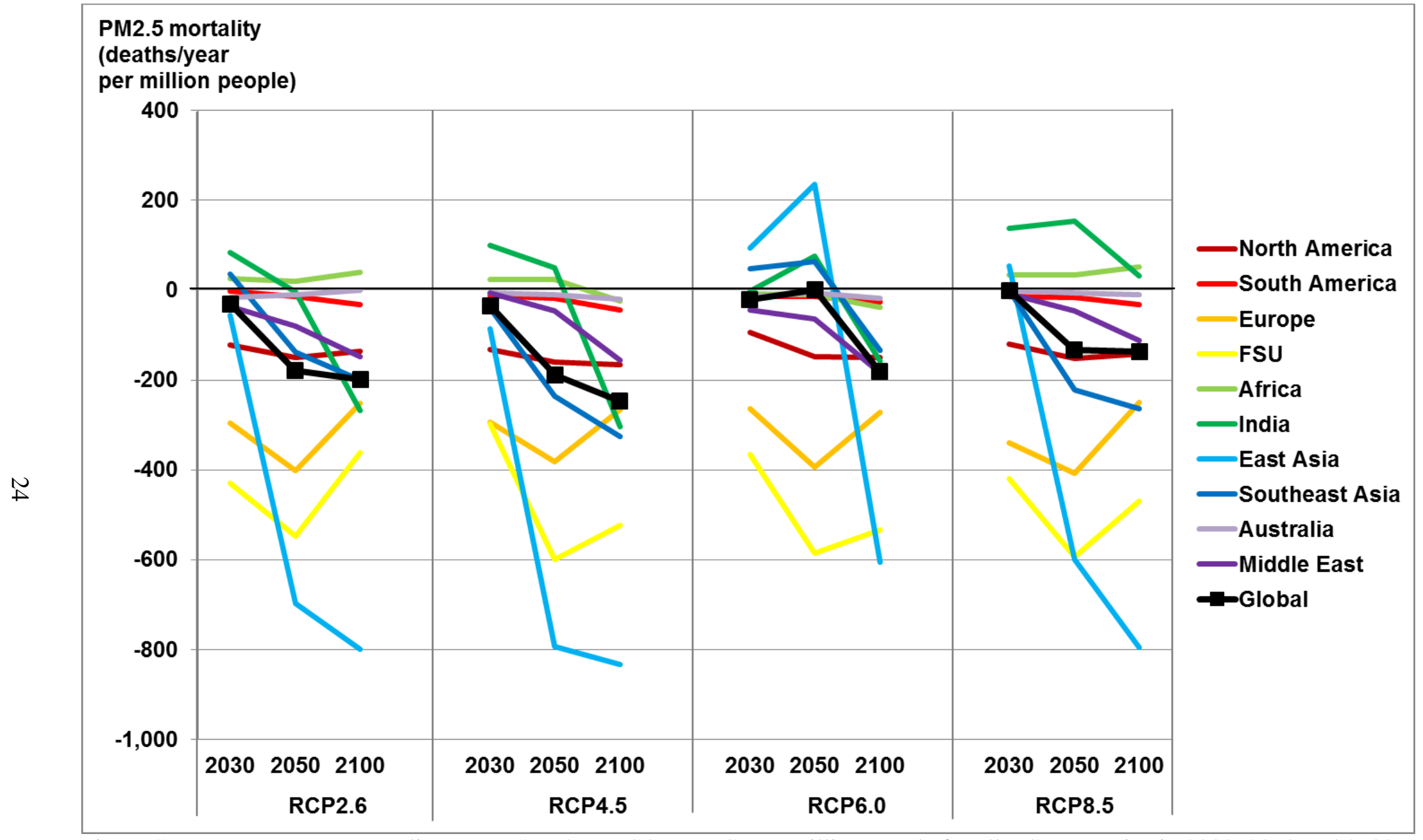

Figure S11 - Future PM 2.5 mortality (IHD+STROKE+COPD+LC) per million people for all RCP scenarios in 2030, 2050 and 2100, showing the multi-model regional average (deaths/year per million people) in ten world regions (Figure S1) and globally, for future air pollutant concentrations relative to 2000 concentrations. 